\title{
Baryon Form Factors at BESIII
}

\author{
Kai $\mathrm{Zhu}^{1, *}$ \\ ${ }^{1}$ Institute of High Energy Physics, Beijing 100049, People's Republic of China
}

\begin{abstract}
In this talk I present recent progress on the studies of baryon form factors at BESIII, including the measurements of the time-like proton electromagnetic form factor via $e^{+} e^{-} \rightarrow p \bar{p}$ in the center-mass-system energy region $2.0-3.08 \mathrm{GeV}$, as well as the electromagnetic form factors of $\Lambda$ and $\Lambda_{c}$, respectively. These results are followed by a short discussion about the prospects of the BESIII program in this area.
\end{abstract}

\section{Introduction}

Proton form factors (FF), electric FF $\left(G_{E}\right)$ and magnetic FF $\left(G_{M}\right)$, are fundamental observable describing the internal structure of proton. They can be extracted via measuring the cross sections for elastic electron-proton scattering in the space-like region $\left(q^{2}<0\right)$ and annihilation processes $e^{+} e^{-} \leftrightarrow p \bar{p}$ in the time-like region $\left(q^{2}>0\right)$. The space-like form factors are assumed to be real, but the time-like form factors can be complex. At low momentum transfer, space-like FFs provide information on the distributions of the electric charges and magnetization within the proton. In the time-like region, electromagnetic FFs can be associated with the time evolution of these distributions, then the quantum chrmodynamics (QCD) scaling is tested. At the high momentum transfer, the size of the nucleus is determined. There are already many measurements of the space-like proton FFs via scattering experiments. At JLab, the ratio was measured precisely with an uncertainty of $1 \%$, based on which the proton electronic and magnetic radii could be extracted. However, the measurements of the timelike form factors are relatively limited. These measurement are performed via $p \bar{p}$ collision or $e^{+} e^{-} \rightarrow p \bar{p}$. The cross section of $e^{+} e^{-} \rightarrow p \bar{p}$ is expressed as following

$$
\frac{d \sigma_{p \bar{p}}(s)}{d \Omega}=\frac{\alpha^{2} \beta C}{4 s}\left[\left|G_{M}\right|^{2}\left(1+\cos ^{2} \theta_{P}\right)+\frac{4 m_{p}^{2}}{s}\left|G_{E}\right|^{2} \sin ^{2} \theta_{p}\right],
$$

where the $\left|G_{M}\right|$ and $\left|G_{E}\right|$ are magnetic and electric form factors, respectively. Lacking accurate data on the angular distributions, most experiments had to assume $\left|G_{M}\right|=\left|G_{E}\right|=\left|G_{\text {eff }}\right|$. There are only two experiments who have measured the ratio $R=\left|G_{E} / G_{M}\right|$, but the results from BaBar [1, 2] and PS170 [3] are inconsistent.

When a u-quark is replaced by a s-quark in a proton, a $\Lambda$ particle is obtained; if a u-quark is replaced by a c-quark in a proton, then a $\Lambda_{c}$ state is obtained. Similar to the FFs of proton, the FFs of $\Lambda$ and $\Lambda_{c}$ take similar functions as they for the proton and can be obtained via measurements of cross sections and angular distributions.

\footnotetext{
*e-mail: zhuk@ihep.ac.cn
} 
The BESIII detector is a general purpose detector [4] with a 93\% solid angle coverage operated at the Beijing Electron Positron Collider (BEPCII). A small-cell helium-based multilayer drift chamber (MDC) determines the momentum of charged particles in a 1 T magnetic field with a resolution of $0.5 \%$ at $1 \mathrm{GeV} / c$, and measures their ionization energy loss $(d E / d x)$ with resolutions better than $6 \%$. A CsI(T1) electromagnetic calorimeter (EMC) measures the photon energies with resolutions $2.5 \%(5.0 \%)$ in the barrel (end caps). A time-of-flight system (TOF), composed of plastic scintillators with resolution of $80 \mathrm{ps}$ (110 ps) in the barrel (end caps), is used for particle identification (PID). A resistive plate chambers based muon counter (MUC) with $2 \mathrm{~cm}$ position resolution is used for muon identification. Since 2009, BESIII has collected the world largest electron positron collision samples at the $\tau$-charm energy region, including $10 \times 10^{9} \mathrm{~J} / \psi$ sample, $5 \times 10^{8} \psi(2 \mathrm{~S})$ sample, $2.9 \mathrm{fb}^{-1} \psi(3770)$ sample, $\sim 1.5 \mathrm{fb}^{-1}$ (150 points) for R and QCD studies (from 2.0 to $4.6 \mathrm{GeV}$ ), and about $13 \mathrm{fb}^{-1}$ data sample for XYZ studies till the talk was presented. These data samples make BESIII suitable to study the time-like form factors of baryons and mesons. In this talk, I shall present recent progress on the studies of time-like form factors of proton, $\Lambda$, and $\Lambda_{c}$ at BESIII.

\section{Proton form factors at BESIII}

Two kinds of data sets are applied in the proton form factors measurement at BESIII. One is the high-energy data sets collected from 3.773 to $4.6 \mathrm{GeV}$, with techniques with tagging the initial state radiation (ISR) photon or un-tagging the ISR photon. The other is low-energy scan data sets collected from 2.0 to $3.08 \mathrm{GeV}$. There is already a publication with the first partial scan data set [5]. The results presented in this talk is based on the full scan data set, that is expected to supersede the previous results.

\subsection{Proton form factors at BESIII with un-tag ISR method}

Comparing to the scan method, ISR technique can provide results at continuous $q^{2}$ range, and the un-tagged ISR analysis would give higher statistics compared with the tagged one. The cross section for the ISR process $e^{+} e^{-} \rightarrow p \bar{p} \gamma$ can be written as

$$
\frac{d \sigma_{e^{+} e^{-} \rightarrow p \bar{p} \gamma}\left(q^{2}\right)}{d q^{2}}=\frac{1}{s} W(s, x) \sigma_{p \bar{p}}\left(q^{2}\right), x=\frac{2 E_{\gamma}^{*}}{\sqrt{s}}=1-\frac{q^{2}}{s}, q^{2}=M_{p \bar{p}}^{2},
$$

where $\alpha$ is the electromagnetic coupling constant, $E_{\gamma}^{*}$ is the energy of the ISR photon in $e^{+} e^{-}$ center mass system, $q$ is the transfer momentum carried by the exchanged virtual photon, the radiation function $W(s, x)$ is the probability of the emission of the hard photon with energy fraction $x, \sigma_{p \bar{p}}\left(q^{2}\right)$ is the cross section without radiative process. The measured cross sections and effective form factors [6] are shown in Fig. 1.

From the measured cross sections and form factors an oscillation is observed, as shown in Fig. 2, via $F_{p}=\left|G_{e f f}\right|-F_{0}$, where $F^{0}$ is the regular behavior over the long range. BESIII confirms the oscillation observed firstly by BaBar [7,8]. It is still unclear this feature is originated from re-scattering effect or resonance contribution or the other physics processes.

The ratio of electric and magnetic forms factors has been obtained in 3 intervals of $M_{p \bar{p}}$ between 2.0 and $3.0 \mathrm{GeV}$. The results, presented in Fig. 3, are consistent with previous measurements.

\subsection{Proton form factors at BESIII with scan data sets}

BESIII has accumulated $688.5 p b^{-1}$ electron positron collision data with 22 energy points from 2.0 to $3.08 \mathrm{GeV}$ center mass energy. With this full low-energy scan data sets, the 



Figure 1. The measured cross sections and form factors via ISR process.
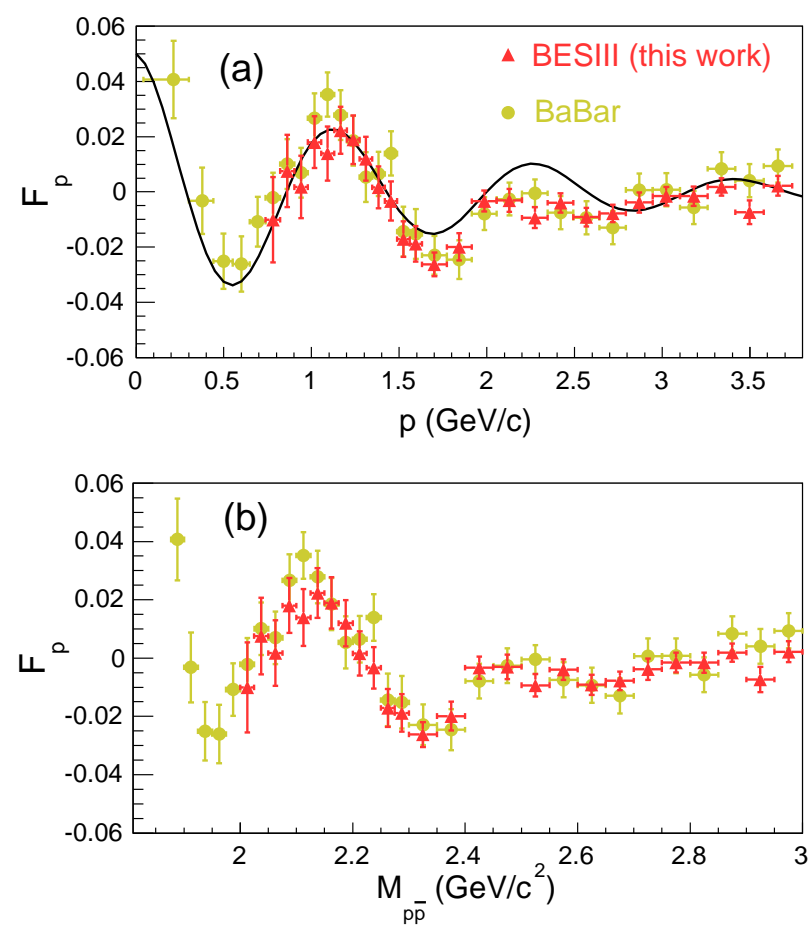

Figure 2. The extracted oscillation feature of the form factors, via ISR process, with respect to (a) momentum and (b) invariant mass.

cross sections of $e^{+} e^{-} \rightarrow p \bar{p}$ are measured, and the form factors are extracted by Eq. 1 . The preliminary results are shown in Fig. 4. In time-like region, BESIII result is an unprecedented accuracy, and the oscillation feature, observed via the ISR method, has been confirmed again. The $R=\left|G_{E} / G_{M}\right|$, shown in Fig. 3, provides an uncertainty comparable to the space-like region for the first time. BESIII measurement of $R=\left|G_{E} / G_{M}\right|$ strongly favors BaBar's result compared to that of PS170. 

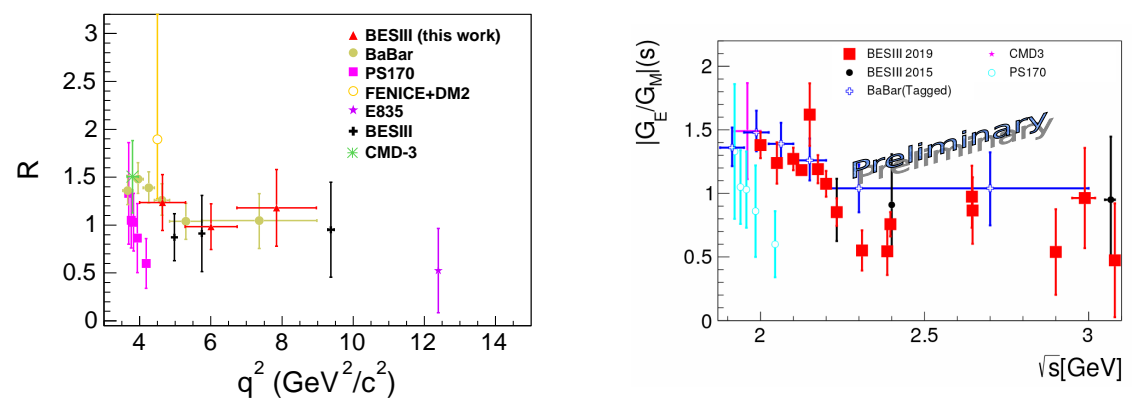

Figure 3. The $R=\left|G_{E} / G_{M}\right|$ of $M_{p \bar{p}}$ between 2.0 and $3.0 \mathrm{GeV}$ via (left) ISR process and (right) scan method, and comparison with other experiments.
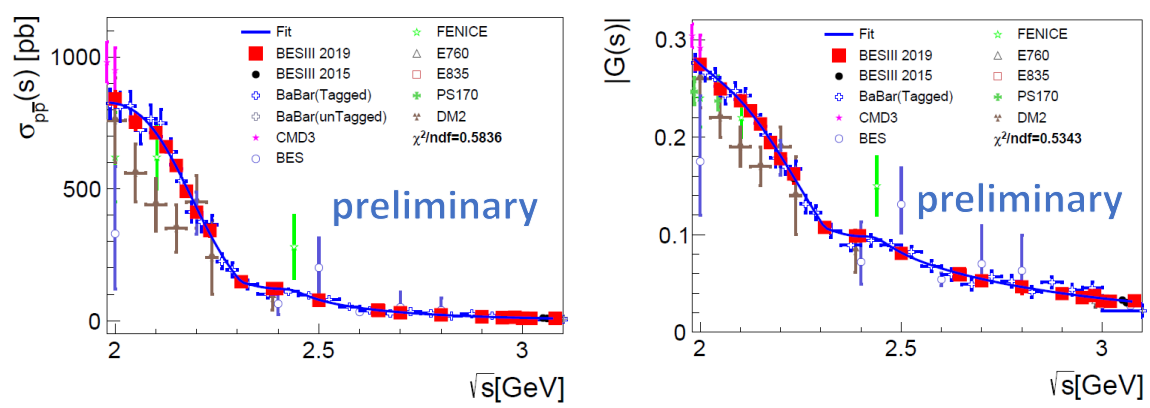

Figure 4. The measured (left) cross sections and (right) form factors via scan method (preliminary).

\section{$3 \Lambda$ form factors at BESIII}

Electromagnetic form factors (EMFFs) are fundamental hadron structure observable describing the deviation of the hyperon to the point-like case since they are related to the chargeand magnetization density. Different to the proton, hyperon is difficult to be studied in the space-like region because it is unstable then the hyperon target is unfeasible, and the quality of hyperon beam is in general not sufficient. So the $e^{+} e^{-}$annihilation offers the best opportunity to study hyper structure. BESIII has measured the cross sections of $e^{+} e^{-} \rightarrow \Lambda \bar{\Lambda}$ in the $\sqrt{s}=2.232,2.400,2.800,3.080 \mathrm{GeV}$, and observed an enhancement near the threshold [9]. Recently BESIII redo the analysis [10] with a joint angular distribution describing the reaction and the hyperons decay chains

$\mathcal{W}(\xi)=\mathcal{T}_{0}+\eta \mathcal{T}_{5}-\alpha_{\Lambda}^{2}\left(\mathcal{T}_{1}+\sqrt{1-\eta^{2}} \cos (\Delta \Phi) \mathcal{T}_{2}+\eta \mathcal{T}_{6}\right)+\alpha_{\Lambda} \sqrt{1-\eta^{2}} \sin (\Delta \Phi)\left(\mathcal{T}_{3}-\mathcal{T}_{4}\right)$

where $\alpha_{\Lambda}$ [11] denotes the decay asymmetry of the $\Lambda \rightarrow p \pi^{-}$decay. The seven functions $\mathcal{T}_{k}(\xi)$ do not depend on the physical quantities $\eta$ and $\Delta \Phi$, but only on the measured angles.

Fig. 5 shows the acceptance corrected $\Lambda$ scattering angle distribution and the product of $\alpha_{\Lambda}$ and $\Lambda$ polarization $P_{y}$ as a function of the scattering angle. The ratio $R=\left|G_{E} / G_{M}\right|$ is determined with unprecedented precision to be $R=0.96 \pm 0.14$ (stat.) \pm 0.02 (sys.). 
The relative phase between $G_{E}$ and $G_{M}$ is determined for the first time to be $\Delta \Phi=$ $37^{\circ} \pm 12^{\circ}$ (stat.) $\pm 6^{\circ}$ (sys.). The non-zero value of the relative phase implies that the EMFFs are complex at this energy. Hence, not only the $s$-wave but also the $d$-wave amplitude contribute to the production.
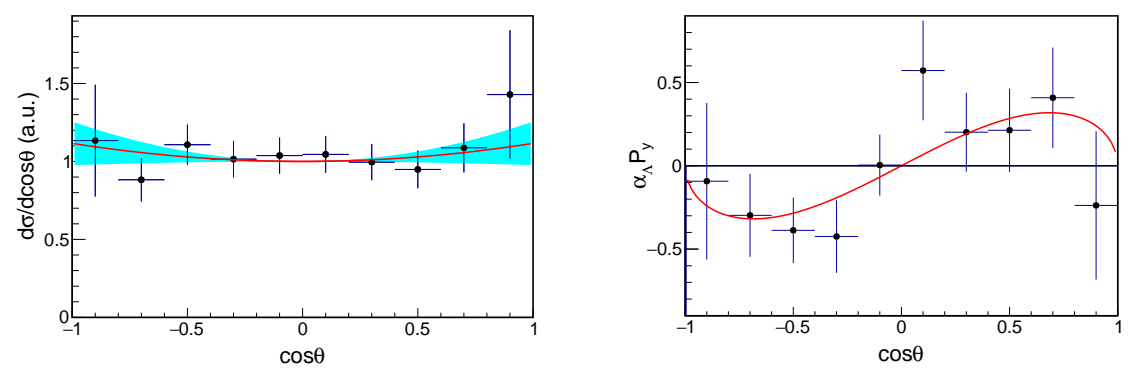

Figure 5. a) The acceptance corrected $\Lambda$ scattering angle distribution. b) The product of $\alpha_{\Lambda}$ and $\Lambda$ polarization $P_{y}$ as a function of the scattering angle.

\section{$4 \Lambda_{c}$ form factors at BESIII}

The cross section of $e^{+} e^{-} \rightarrow \Lambda_{c}^{+} \bar{\Lambda}_{c}^{-}$near threshold are measured at $\sqrt{s}=4574.5,4580.0$, 4590.0, 4559.5 MeV [12]. From Fig. 6 it can be seen the trend of BESIII is different to that of Belle or phase space. The ratio $R=\left|G_{E} / G_{M}\right|$ are measured to be $1.14 \pm 0.14 \pm 0.07$ and $1.23 \pm 0.05 \pm 0.03$ at the energy points $\sqrt{s}=4574.5 \mathrm{MeV}$ and $4599.5 \mathrm{MeV}$, respectively.

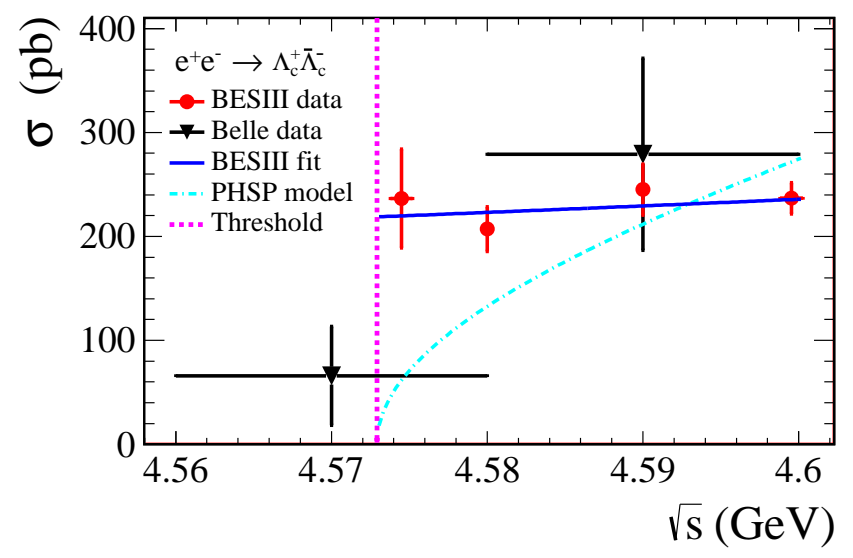

Figure 6. Cross section of $e^{+} e^{-} \rightarrow \Lambda_{c}^{+} \bar{\Lambda}_{c}^{-}$by BESIII and Belle.

\section{Summary and outlook}

Proton form factors have been measured at BESIII via both ISR and scan technique. The results are in significantly improved precision and some novel/unexpected features are observed/confirmed. $\Lambda$ form factors with relative phase between $G_{E}$ and $G_{M}$ are measured for 
the first time. The effective form factors and $\left|G_{E} / G_{M}\right|$ of $\Lambda_{c}$ have been measured near the threshold. BESIII will continue to measure the proton form factors with varied technique, especially at the region very close to the threshold. And with the large data samples collected at the $\tau$-charm region, form factors of other baryons, such as neutron, $\Omega, \Sigma, \Xi$, as well as their polarizations will be measured soon. The measurements of $e^{+} e^{-}$annihilating into $\Lambda_{c}$ pair will be performed at higher energy points to check the consistence with Belle and study the $\mathrm{Y}(4660)$ state.

\section{Acknowledgement}

I am sincerely grateful to the PhiPsi2019 committee for the organization of this wonderful conference.

\section{References}

[1] J. P. Lees et al. [BaBar Collaboration], Phys. Rev. D 88, 032011 (2013) [arXiv:1306.2895 [hep-ex]].

[2] J. P. Lees et al. [BaBar Collaboration], Phys. Rev. D 87, no. 9, 092005 (2013) [arXiv:1302.0055 [hep-ex]].

[3] G. Bardin et al., Nucl. Phys. B 411, 3 (1994).

[4] M. Ablikim et al. (BESIII Collaboration), Nucl. Instrum. Meth. A 614, 345 (2010).

[5] M. Ablikim et al. [BESIII Collaboration], Phys. Rev. D 91, no. 11, 112004 (2015) [arXiv:1504.02680 [hep-ex]].

[6] M. Ablikim et al. [BESIII Collaboration], arXiv:1902.00665 [hep-ex].

[7] A. Bianconi and E. Tomasi-Gustafsson, Phys. Rev. Lett. 114, no. 23, 232301 (2015) [arXiv:1503.02140 [nucl-th]].

[8] A. Bianconi and E. Tomasi-Gustafsson, Phys. Rev. C 93, no. 3, 035201 (2016) [arXiv:1510.06338 [nucl-th]].

[9] M. Ablikim et al. [BESIII Collaboration], Phys. Rev. D 97, no. 3, 032013 (2018) [arXiv:1709.10236 [hep-ex]].

[10] M. Ablikim et al. [BESIII Collaboration], arXiv:1903.09421 [hep-ex].

[11] M. Ablikim et al. [BESIII Collaboration], arXiv:1808.08917 [hep-ex].

[12] M. Ablikim et al. [BESIII Collaboration], Phys. Rev. Lett. 120, no. 13, 132001 (2018) [arXiv:1710.00150 [hep-ex]]. 